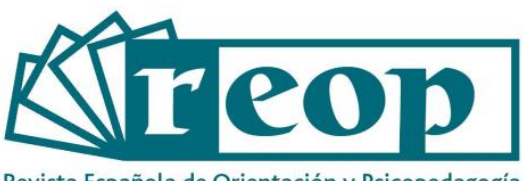

Revista Española de Orientación y Psicopedagogía

\title{
DIFICULTADES EN EL AUTOCONCEPTO EN FUNCIÓN DEL SEXO Y ESTUDIOS DE JÓVENES CON RETINOSIS PIGMENTARIA
}

\section{SELF-CONCEPT DIFFICULTIES ACCORDING TO SEX AND STUDIES OF YOUNG PEOPLE WITH RETINITIS PIGMENTOSA}

\begin{abstract}
Helena Chacón-López ${ }^{1}$
Universidad de Granada. Facultad de Ciencias de la Educación. Departamento de Psicología Evolutiva
\end{abstract} y de la Educación. Granada, España

María-Dolores López-Justicia

Universidad de Granada. Facultad de Psicología. Departamento de Psicología Evolutiva y de la Educación. Granada, España

\section{RESUMEN}

Introducción: Dada la relevancia del autoconcepto en el ámbito educativo y en la vida personal, social y laboral, en la presente investigación se analizan intra-sexos las dificultades en este constructo en jóvenes de 18 a 30 años (14 hombres y 23 mujeres) con degeneración retiniana, frente a otros sin afectación; indagando en el papel que desempeña el nivel de estudios (primarios/secundarios,

1 Correspondencia: Dra. Helena Chacón-López, Universidad de Granada, Departamento de Psicología Evolutiva y de la Educación, Facultad de Ciencias de la Educación, Campus Universitario de la Cartuja, s/n, 18071, Granada (España). Teléfono: (0034)958243975. E-mail: helenachacon@ugr.es 
universitarios). Método: Participaron 74 personas (37 afectados y 37 sin afectación), igualados en sexo y nivel educativo. Se utilizó la Escala de Autoconcepto Tennesse y un cuestionario sociodemográfico elaborado ad hoc. Resultados: Los resultados obtenidos en los distintos análisis no paramétricos llevados a cabo mostraron que, tanto entre mujeres como entre hombres afectados, se detectaron puntuaciones inferiores en autoconcepto físico; constatándose igualmente que las mujeres con estudios superiores se percibían mejor en éste; mientras que los hombres obtenían mayores puntuaciones en el factor personal. Conclusiones: Se sugiere a los profesionales de la orientación y psicopedagogía que trabajan con estos jóvenes identificar los puntos fuertes en los que cada cual destaque reforzándolos y resaltándolos, así como detectar los aspectos más deficitarios del autoconcepto para entrenarlos, en aras de favorecer el sentimiento de valía y competencia personal.

Palabras clave: Educación, orientación, sexo, autoconcepto, retinosis pigmentaria.

\section{ABSTRACT}

Introduction: Attending to the relevance of self-concept in the educational field and in the personal, social and labor life, this study analyzed the difficulties intra-sexes of young people between 18 to 30 years old (14 men y 23 women) affected by a retinal degeneration, compared to others without affectation; enquire into the role of the academic level (primary/secondary, university). Method: Participants were 74 people (37 affected and 37 non-affected), matched in sex and educational level. The Tennessee Self-Concept Scale and a sociodemographic questionnaire elaborated ad hoc were used. Results: The results obtained in the different non-parametric analysis carried out, between affected women and between affected men, showed that lower scores were detected in physical self-concept; verifying that women with higher studies were perceived better in this; while men obtained higher scores in personal selfconcept. Conclusions: Data suggested to the guidance and psychopedagogy professionals who work with these young people to identify the strong points in which each one stands out, reinforcing and highlighting them, as well as detecting the most deficient aspects of the self-concept in order to train them, in order to promote the feeling of worth and personal competence.

Key Words: Education, guidance, sex, self-concept, retinitis pigmentosa.

\section{Introducción}

Las repercusiones psicológicas, educativas y sociales que tienen las enfermedades crónicas degenerativas de retina sobre la calidad de vida de adolescentes y jóvenes que las presentan son indudables, al implicar diversos grados de limitación funcional asociadas al avance de la patología y grado de afectación (Chacón-López y López-Justicia, 2016; Fernández, 2007; Rundquist, 2004). Un ejemplo de estas enfermedades es la retinosis pigmentaria (RP), problemática que produce una paulatina y grave disminución de la función visual (Fernández, 2007; Rundquist, 2004) y que presenta características clínicas como: afectación de los dos ojos, pérdida de visión periférica o central; dificultad para ver de noche, o en ambientes con poca luz; dificultad de adaptación a los cambios de luz; o alteraciones en la discriminación de colores (Rundquist, 2004). Su incidencia es variable, aunque es algo más frecuente en hombres que en mujeres (Fernández, 2007). 
El avance y progresión de esta enfermedad es impredecible conllevando una sucesiva pérdida de habilidad funcional para realizar determinadas tareas de la vida diaria, o de desenvolvimiento en el espacio (Fuhr, Liu, y Kuyk, 2007; Rundquist, 2004), provocando que muchas personas afectadas se perciban más negativamente en estos ámbitos (Kiser y Dagnelie, 2008). Hasta el momento no existen soluciones médicas o tratamientos farmacológicos que resuelvan los efectos de esta patología, al igual que tampoco existen para otras problemáticas visuales con carácter degenerativo. Además, debido al carácter progresivo y degenerativo, generalmente, los efectos no son manifiestos hasta estadios avanzados, de ahí que el grupo de afectados forme parte del colectivo de personas con discapacidad no visible (que incluye a aquellas con una serie de condiciones físicas y psicológicas que pueden o no presentar manifestaciones visibles de su discapacidad), pudiendo provocarles dificultades en el ámbito psicológico, educativo y social (Zapata, 2018).

Aunque los síntomas de la patología suelen iniciarse en la infancia, es alrededor de los 20 años cuando el trastorno empieza a ser más incapacitante visualmente en un buen número de personas (Chacón-López, López-Justicia, Fernández, Chacón, y Polo, 2013; Fernández, 2007; López-Justicia, Polo, Fernández, Chacón-López, Díaz-Batanero, y Chacón, 2011), época en la que, por regla general, comienzan a orientarse en su futuro académico, profesional y personal. Es conocido que los procesos de transición a otras etapas educativas son de gran relevancia para el alumnado (Fernández Sierra y Santos-Bocero, 2014), pudiendo tener una repercusión mayor en estos jóvenes que deben enfrentarse no solo a las consecuencias de una enfermedad progresiva cuyos síntomas pueden influir considerablemente en su educación, movilidad, socialización y empleo (Chacón-López, López-Justicia, y Vervloed, 2014); sino a los cambios que conlleva el acceso a otros niveles educativos. Ello asocia frecuentemente la aparición de distintos problemas psicológicos, tales como alteraciones en el autoconcepto (Chacón-López y López-Justicia, 2016; Fernández, López-Justicia, y Polo, 2007; LópezJusticia y Nieto 2006; López-Justicia et al., 2011), en la autoeficacia percibida (Zapata, 2018), o en el estado emocional (López-Justicia y Chacón-López, 2015; López-Justicia, Polo, et al., 2011; Moschos, Chatzirallis, y Chatzirallis, 2015).

El interés en el estudio del autoconcepto en esta población radica en que es un indicador de bienestar psicológico y satisfacción personal que contribuye al equilibrio mental y a la salud en general (Fuentes, García, Gracia, y Lila, 2011; Goñi, Esnaola, Rodríguez, y Camino, 2015). Asimismo, tiene importantes implicaciones para el desarrollo de una vida positiva, siendo una variable significativa en el logro de objetivos y en el adecuado desarrollo en la sociedad, en todos los ámbitos (Halder y Datta, 2012). En personas con discapacidad se han relacionado niveles altos de autoconcepto con la superación de sus consecuencias, con mayor participación y compromiso social, con una actitud positiva ante la vida, etc.; mientras que bajos niveles repercuten negativamente en la salud física y psíquica y se relacionan con infravaloración personal, depresión, aislamiento, etc. (Smith, NolenHoeksema, Fredrickson, y Loftus, 2003), así como con el desarrollo de las habilidades sociales (Jessup, Bundy, Hancock, y Broom, 2018).

El autoconcepto se refiere al conjunto de características que cada persona utiliza para definirse como individuo y diferenciarse de los demás (Harter, 1999), estando integrado por: la percepción que una persona tiene de sí misma (autoconcepto propiamente dicho o autoimagen); el valor que atribuye a su particular manera de percibirse (la autoestima); y su comportamiento, ya que el autoconcepto influye en la conducta y la condiciona en gran medida. Asimismo, tiene un carácter jerárquico y multidimensional (González-Pienda et al., 2003) y evoluciona con los años como resultado de la influencia sociocultural, educativa y experiencia personal en su medio social (Manitsa, Barlow-Brown y Lyons, 2019), aceptándose que mientras el autoconcepto general puede mantenerse prácticamente inalterable (Coll, 2002), los más específicos pueden variar. 

Autoconcepto y relación con el sexo y nivel de estudios en personas con discapacidad visual
manteniendo resto de visión

En el II Plan de Acción Nacional para las personas con Discapacidad (2003-2007), así como en el III Plan (2009-2012), se recoge la meta de lograr su integración en el sistema educativo y la educación a lo largo del ciclo vital, ofreciendo la respuesta más adecuada a las necesidades que presenta cada estudiante y garantizando de este modo no solo el acceso a los diferentes niveles educativos, sino la permanencia en éstos de las personas que presentan alguna discapacidad. Metas que también aparecen incluidas en el III Plan de la comunidad de Madrid (2012-2015) (Simón Rueda, FernándezBlázquez, Pérez-de la Merced, Márquez Vázquez, Echeita Sarrionandia y Moreno Hernández, 2019). A pesar de este propósito, en España no ha sido frecuente hasta hace pocos años que personas con discapacidad accedieran a estudios medios o universitarios, siendo del $1.5 \%$ el porcentaje de estudiantes universitarios con discapacidad censados en el año 2018 (Fundación Universia y CERMI, 2018). Sin embargo, mayor formación académica suele ir asociada a mayor capacidad, competencia, oportunidades, habilidades, etc., convirtiéndose el acceso a estudios superiores en una experiencia favorecedora del sentimiento de valía personal (López-Justicia et al., 2011).

Partiendo de la premisa de que la educación no solo es un derecho, sino una oportunidad para mujeres y hombres con discapacidad, diversas investigaciones han indagado en el papel que desempeña el sexo y el nivel educativo en el autoconcepto de población afectada por dificultades visuales. Así, se ha puesto de manifiesto diferencias ligadas al sexo en el autoconcepto de adolescentes con discapacidad visual (Al-Zyoudi, 2007; Augestad, 2017b; López-Justicia y Pichardo, 2001), aunque otros no han hallado diferencias significativas (Datta y Talukdar, 2016; Garaigordobil y Bernarás, 2009; Mishra y Singh, 2012), pero sí constataron que las chicas tenían puntuaciones más bajas en autoestima, variable vinculada estrechamente con el autoconcepto (Garaigordobil y Bernarás, 2009), y presentaban síntomas depresivos con mayor frecuencia (Augestad, 2017a). Otro estudio llevado a cabo entre personas afectadas por RP con un rango muy amplio de edad (entre 19 y 60 años), detectó que las mujeres se percibían mejor que los hombres en el factor/dimensión física y que aquellas con un nivel más alto de estudios puntuaban más positivamente en este factor o dimensión (LópezJusticia et al., 2011). Mientras que Chacón-López y López-Justicia (2016), constataron que las personas con RP que presentaban mayor sintomatología depresiva obtenían puntuaciones más bajas en todos los factores del autoconcepto, siendo las mujeres quienes puntuaban más bajo en todos ellos.

\section{Objetivos del estudio}

Hace ya algunos años que se puso de manifiesto la relevancia del autoconcepto en la competencia académica de los jóvenes, al influir en el rendimiento educativo, social y laboral, y contribuir a la salud y equilibrio psíquico (Fuentes et al., 2011; Goñi et al., 2015). De ahí que también se haya prestado cierta atención a la repercusión que tiene el nivel de estudios en el autoconcepto de personas con RP, pero no se han encontrado investigaciones que traten de conocer las dificultades que presentan mujeres y hombres jóvenes afectados (con edades situadas entre 18 y 30 años), frente a iguales sin alteraciones visuales; ni el papel que desempeña el nivel de estudios (primarios/secundarios, universitarios). El interés por centrarse en esta época de la vida se justifica por ser una etapa en la que mujeres y hombres deben tomar decisiones importantes acerca de su futuro siendo, generalmente, ya manifiestos 
los efectos de la degeneración retiniana. Estos efectos podrían repercutir negativamente en el autoconcepto, dado su papel en la vida personal, educativa y emocional de esta población (ChacónLópez y López-Justicia, 2016).

El análisis intra-sexos del estado del autoconcepto, y conocer la influencia que desempeña el nivel de estudios, son aspectos a considerar en la orientación psicopedagógica y en la posterior planificación de la actuación educativa, laboral y social con hombres y mujeres afectados; habida cuenta de sus dificultades asociadas a la restricción visual. Los resultados obtenidos pueden aportar alguna luz que ayude a estos profesionales en su actuación con un colectivo muchas veces olvidado, dado su carácter minoritario; lo que no excusa la escasez de investigación en la temática. Son razones que justifican el

presente trabajo, cuyo primer objetivo es conocer si existen diferencias entre mujeres con RP frente a mujeres sin ella en algún factor del autoconcepto; así como entre hombres con RP frente a otros sin afectación. La hipótesis de partida es que la problemática visual puede determinar diferencias en algún factor del autoconcepto, esperando hallar puntuaciones más bajas en algún factor del mismo en el grupo de mujeres y hombres con RP.

El segundo objetivo trata de dilucidar la existencia de diferencias intrasexos en el grupo de población con RP, asociadas al nivel de estudios (primarios y secundarios/ universitarios), en algún factor del autoconcepto. Se prevé que existan diferencias en función del nivel de estudios tanto en el grupo de mujeres como en el de hombres con RP; esperando encontrar puntuaciones más bajas en algún factor del autoconcepto en quienes poseen menor nivel de estudios.

\section{Método}

\section{Participantes}

Participaron un total de 74 personas (28 hombres y 46 mujeres), 37 con RP (14 hombres y 23 mujeres) y 37 personas sin afectación (14 hombres y 23 mujeres), con edades comprendidas entre 18 y 30 años. El grupo de personas afectadas por RP participaban voluntariamente en un proyecto coordinado por una de las autoras, y eran miembros de dos Asociaciones españolas. Fueron seleccionados por conveniencia entre los que mantenían resto visual y que cumplían los siguientes requisitos: padecer degeneración retiniana, pero manteniendo resto visual; haber sido diagnosticada la enfermedad al menos 3 años antes de la evaluación (para tener la garantía de haber transcurrido un tiempo suficiente tras el diagnóstico y evitar el efecto que éste pudiera tener en las respuestas); y no presentar otro tipo de enfermedad o alteración sensorial.

Los años transcurridos desde el diagnostico oscilaban entre 3 y 15 años $(M=14.58$ y $D T=11.135)$ y sus características socio-demográficas eran las siguientes: edad entre 18 y 30 años $(M=25.9$ y $D T=$ 5.997), 30 eran solteros, 7 estaban casados (3 hombres y 4 mujeres). Entre los hombres: 6 tenían estudios primarios o secundarios y 8, superiores. Entre las mujeres: 10 tenían estudios primarios 0 secundarios y 13 superiores. La situación laboral del grupo era: 20 estudiantes, 9 desempleados y 8 trabajadores. El grupo de no afectados fueron seleccionados al azar entre una muestra de 110 jóvenes (70 mujeres y 40 hombres), equiparados por edad $(M=26.5$ y $D T=6.12$ ) y nivel de estudios al grupo de 
RP. Entre los hombres, 5 tenían estudios primarios/secundarios y 9 superiores. Entre las mujeres, 12 con estudios primarios/secundarios y 11 poseían estudios superiores.

\section{Instrumentos}

Se utilizó la Escala de Autoconcepto Tennesse (T.S.C.S.) (Fitts, 1965), adaptada y validada por Garanto (1984) para población española. Se eligió por haber sido usada en otros estudios con personas de características similares a las de éste y por obtener datos relativos a la estructura multidimensional del autoconcepto, aspecto considerado esencial en la actualidad. Los datos de la fiabilidad, obtenida mediante test-retest, son adecuados (.87 para el factor físico, .80 para el factor moral-ética, .85 para el factor personal, .89 para el factor familiar y .90 para el factor social). La validez concurrente (calculada mediante correlación de Pearson) mostraba correlaciones altamente significativas, indicativas de fuerte consistencia interna.

La escala está compuesta por 100 ítems/preguntas (con respuesta tipo Likert de 5 puntos: 1, indica completamente falso; 5 , indica completamente cierto), 45 expresadas en forma positiva y 45 en forma negativa, las 10 preguntas restantes pertenecen a la escala $L$ (Mentira) del Inventario Multifásico de Personalidad de Minnesotta (Minnesota Multiphasic Personality Inventory - MMPI), que está relacionado con la autocrítica. Los 90 ítems aparecen clasificados en dos dimensiones, una interna: consta de tres escalas: a) Identidad o autoconcepto; b) Autosatisfacción o autoestima; y c) Autocomportamiento); y otra externa que consta de cinco escalas o factores: Personal (sentido de los valores interiores, sentimiento de adecuación como persona, valoración de su personalidad); Familiar (valoración e importancia como miembro de su familia y perteneciente a un círculo restringido de amigos); Moral-ético (fuerza moral o ética, convicción de ser una buena persona y atractiva moralmente); Social (capacidad de adecuación y valor de la interacción social con otras personas); y Físico (cómo inciden en su persona todos sus elementos físicos, con qué intensidad y direccionalidad; valoración de su apariencia física). La máxima puntuación que se puede obtener en el factor personal oscila entre 46 y $84(M=65)$ para hombres y entre 46 y $78(M=65)$ para mujeres; en el físico, entre 41 y $82(M=67)$ para los primeros y entre 47 y $80(M=68)$ para las segundas; en el moral/ético, entre 45 y $82(M=69)$ para hombres y entre 55 y $82(M=70)$ para las mujeres; el familiar, entre 43 y $87(M=67)$ para los hombres y entre 54 y $82(M=71)$ para las mujeres; el social, entre 50 y $82(M=65)$ para los hombres y entre 50 y $85(M=67)$ para las mujeres. El tiempo en que se puede completar la prueba está entre 13 y 20 minutos.

Se administró un segundo cuestionario, elaborado ad hoc, para obtener información sobre variables sociodemográficas (edad, años desde el diagnóstico, nivel de estudios, estado civil y situación laboral).

\section{Procedimiento}

Tras convocar a las personas afectadas (participantes en un proyecto coordinado por una de las autoras), se les informó sobre los objetivos del estudio y los requisitos a cumplir, solicitándose su participación voluntaria a todos los que los cumplían. Tras seleccionar la muestra por conveniencia, proporcionaron un informe oftalmológico, que incluía el diagnóstico y demás características clínicas. Seguidamente, cada uno de los afectados cumplimentó la ficha personal y posteriormente el 
cuestionario de autoconcepto. Los cuestionarios fueron debidamente ampliados al tamaño de letra que eran capaces de ver, entre 14 y 16 puntos.

Con el fin de seleccionar un grupo control se convocó a alumnado de último curso del grado de Psicología, para que voluntariamente evaluaran a hombres y mujeres que, distribuidos homogéneamente por edad y nivel de estudios, no presentaran ninguna afectación visual y no tuvieran contacto con esta problemática. El alumnado fue entrenado y supervisado en la administración de las pruebas y ante cualquier indicio de duda sobre una correcta evaluación, éstas eran rechazadas. Se seleccionó al azar una muestra entre las personas evaluadas ( $\mathrm{N}=110$ : 70 mujeres y 40 hombres).

Todos firmaron el consentimiento informado elaborado para la investigación, aprobado por el Comité de Ética de la Universidad de Granada.

\section{Análisis de los datos}

El análisis de los datos fue realizado usando el paquete estadístico SPSS versión 20.0. para Windows. Primeramente, se buscaron diferencias intra-sexos en los distintos factores considerados sobre autoconcepto, entre mujeres con RP frente a mujeres sin ella, aplicando la prueba de Mann-Whitney (debido al tamaño reducido de la muestra). A continuación, se llevó a cabo una comparación (aplicando la misma prueba) de acuerdo con el nivel de estudios en los distintos factores de autoconcepto; primeramente, en el grupo de mujeres afectadas por RP y, seguidamente, otra comparación en el grupo de mujeres no afectadas. En ambos casos se dividió la muestra entre participantes con estudios primarios o medios y participantes con estudios superiores o universitarios.

Idéntico procedimiento y análisis se siguió en el grupo de hombres con RP frente a hombres sin afectación, en relación con los distintos factores de autoconcepto considerados.

\section{Resultados}

Los resultados de las comparaciones entre mujeres con RP frente a mujeres sin ella (ver Tabla 1), en relación con los diferentes factores de autoconcepto reflejaron diferencias significativas (de acuerdo con la prueba de Mann-Whitney), en el factor físico $(U=32.50 ; p=0.02)$, mostrando puntuaciones más bajas aquéllas con RP.

En el resto de factores no hubo diferencias significativas entre los grupos, aunque se constataron peores puntuaciones en mujeres con RP (Tabla 1).

Al analizar el nivel de estudios en el grupo de mujeres con RP se hallaron diferencias nuevamente en el factor físico a favor de aquellas con estudios universitarios $(U=39.00 ; p=0.24)$, frente a las que tenían estudios primarios y medios, siendo las primeras quienes puntuaban más alto.

En el grupo de mujeres sin afectación no se hallaron diferencias en ningún factor (Tabla 2). 
Tabla 1

Puntuaciones medias, desviaciones típicas y diferencias en factores de autoconcepto entre mujeres con $R P$ y $\sin R P$

\begin{tabular}{llcrcc}
\hline \multicolumn{1}{c}{ Variable } & \multicolumn{1}{c}{ Grupos } & M & DT & $\begin{array}{c}\text { U de Mann- } \\
\text { Whitney }\end{array}$ & p \\
\hline \multirow{2}{*}{ Autoconcepto Físico } & Mujeres con RP & 60.81 & 7.46 & 32.50 & .02 \\
& Mujeres sin RP & 66.83 & 3.81 & & \\
\multirow{2}{*}{ Autoconcepto Moral-Ético } & Mujeres con RP & 65.27 & 6.87 & 59.00 & .35 \\
& Mujeres sin RP & 69.33 & 8.01 & & \\
Autoconcepto Personal & Mujeres con RP & 64.88 & 7.07 & 69.00 & .66 \\
& Mujeres sin RP & 65.33 & 8.64 & & \\
Autoconcepto Familiar & Mujeres con RP & 68.50 & 6.61 & 59.50 & .35 \\
\multirow{2}{*}{ Autoconcepto Social } & Mujeres sin RP & 70.33 & 8.61 & & \\
& Mujeres con RP & 65.81 & 6.95 & \multirow{2}{*}{44.50} & .10 \\
\hline
\end{tabular}

Nota: Diferencia significativa en $p<.05$

Tabla 2

Puntuaciones medias, desviaciones típicas y diferencias según nivel de estudios en factores de autoconcepto grupo mujeres con RP y sin RP

\begin{tabular}{|c|c|c|c|c|c|c|c|c|c|}
\hline \multirow[b]{2}{*}{ Variable } & \multirow[b]{2}{*}{$\begin{array}{l}\text { Grupos (por } \\
\text { estudios) }\end{array}$} & \multicolumn{4}{|c|}{ Mujeres con RP } & \multicolumn{4}{|c|}{ Mujeres sin RP } \\
\hline & & M & DT & $\begin{array}{c}\text { U de } \\
\text { Mann- } \\
\text { Whitney }\end{array}$ & $\mathbf{p}$ & $\mathbf{M}$ & DT & $\begin{array}{c}\text { U de } \\
\text { Mann- } \\
\text { Whitney }\end{array}$ & $\mathbf{p}$ \\
\hline \multirow{2}{*}{$\begin{array}{l}\text { Autoconcepto } \\
\text { Físico }\end{array}$} & $\begin{array}{l}\text { Primarios y } \\
\text { Secundarios }\end{array}$ & 57.67 & 4.76 & \multirow{2}{*}{39.00} & \multirow{2}{*}{.02} & 60.32 & 8.42 & \multirow{2}{*}{94.50} & \multirow{2}{*}{.27} \\
\hline & Universitarios & 65.09 & 8.51 & & & 64.46 & 6.59 & & \\
\hline \multirow{2}{*}{$\begin{array}{l}\text { Autoconcepto } \\
\text { Moral-Ético }\end{array}$} & Primarios y & 63.67 & 7.32 & \multirow{2}{*}{59.50} & \multirow{2}{*}{.23} & 66.79 & 5.66 & \multirow{2}{*}{122.50} & \multirow{2}{*}{.67} \\
\hline & Universitarios & 67.45 & 5.82 & & & 65.62 & 8.27 & & \\
\hline \multirow{2}{*}{$\begin{array}{l}\text { Autoconcepto } \\
\text { Personal }\end{array}$} & $\begin{array}{l}\text { Primarios y } \\
\text { Secundarios }\end{array}$ & 64.07 & 7.20 & \multirow{2}{*}{78.50} & \multirow{2}{*}{.83} & 65.11 & 7.63 & \multirow{2}{*}{101.00} & \multirow{2}{*}{.40} \\
\hline & Universitarios & 66.60 & 7.08 & & & 66.69 & 7.39 & & \\
\hline \multirow{2}{*}{$\begin{array}{l}\text { Autoconcepto } \\
\text { Familiar }\end{array}$} & $\begin{array}{l}\text { Primarios y } \\
\text { Secundarios }\end{array}$ & 67.27 & 6.07 & \multirow{2}{*}{56.50} & \multirow{2}{*}{.18} & 68.11 & 5.62 & \multirow{2}{*}{105.00} & \multirow{2}{*}{.49} \\
\hline & Universitarios & 70.18 & 7.23 & & & 69.23 & 5.74 & & \\
\hline \multirow{2}{*}{$\begin{array}{l}\text { Autoconcepto } \\
\text { Social }\end{array}$} & $\begin{array}{l}\text { Primarios y } \\
\text { Secundarios }\end{array}$ & 64.60 & 5.61 & \multirow{2}{*}{62.50} & \multirow{2}{*}{.30} & 63.32 & 8.18 & \multirow{2}{*}{85.50} & \multirow{2}{*}{.14} \\
\hline & Universitarios & 67.45 & 8.45 & & & 67.77 & 8.16 & & \\
\hline
\end{tabular}

Nota: Diferencia significativa en $p<.05$ 
Los resultados de las comparaciones entre hombres con RP frente a hombres sin afectación (Tabla $3)$, reflejaron diferencias significativas en el factor físico $(U=17.50 ; p=0.001)$, siendo aquéllos con $\mathrm{RP}$ los que obtenían puntuaciones más bajas.

En el resto de factores no hubo diferencias significativas entre los grupos, aunque se constataron peores puntuaciones en los hombres con RP en todos los factores (ver Tabla 3).

\section{Tabla 3}

Puntuaciones medias, desviaciones típicas y diferencias en factores de autoconcepto entre hombres con RP y sin RP

\begin{tabular}{lllccc}
\hline \multicolumn{1}{c}{ Variable } & \multicolumn{1}{c}{ Grupos } & M & DT & $\begin{array}{c}\text { U de Mann- } \\
\text { Whitney }\end{array}$ & p \\
\hline \multirow{2}{*}{ Autoconcepto Físico } & Hombres con RP & 56.62 & 6.72 & 17.50 & .001 \\
& Hombres sin RP & 67.77 & 4.58 & & \\
\multirow{2}{*}{ Autoconcepto Moral-Ético } & Hombres con RP & 67.46 & 3.09 & 63.50 & .27 \\
& Hombres sin RP & 68.92 & 5.28 & & \\
Autoconcepto Personal & Hombres con RP & 65.85 & 5.98 & 57.00 & .15 \\
& Hombres sin RP & 69.23 & 4.54 & & \multirow{2}{*}{ Autoconcepto Familiar } \\
\cline { 2 - 5 } Autoconcepto Social & Hombres con RP & 67.62 & 6.51 & 71.50 & .51 \\
& Hombres sin RP & 69.15 & 5.12 & & \multirow{2}{*}{.71} \\
\hline
\end{tabular}

Nota: Diferencia significativa en $p<.05$

\section{Tabla 4}

Puntuaciones medias, desviaciones típicas y diferencias según nivel de estudios en factores de autoconcepto en grupos de hombres con RP y sin RP

\begin{tabular}{|c|c|c|c|c|c|c|c|c|c|}
\hline \multirow[b]{2}{*}{ Variable } & \multirow[b]{2}{*}{$\begin{array}{l}\text { Grupos (por } \\
\text { estudios) }\end{array}$} & \multicolumn{4}{|c|}{ Hombres con RP } & \multicolumn{4}{|c|}{ Hombres sin RP } \\
\hline & & $\mathbf{M}$ & DT & $\begin{array}{c}\text { U de } \\
\text { Mann- } \\
\text { Whitney }\end{array}$ & $\mathbf{p}$ & $\mathbf{M}$ & DT & $\begin{array}{c}\text { U de } \\
\text { Mann- } \\
\text { Whitney }\end{array}$ & $\mathbf{p}$ \\
\hline \multirow{2}{*}{$\begin{array}{l}\text { Autoconcepto } \\
\text { Físico }\end{array}$} & $\begin{array}{l}\text { Primarios y } \\
\text { Secundarios }\end{array}$ & 60.20 & 8.18 & \multirow{2}{*}{61.00} & \multirow{2}{*}{.33} & 68.50 & 4.20 & \multirow{2}{*}{15.50} & \multirow{2}{*}{.71} \\
\hline & Universitarios & 63.44 & 7.89 & & & 67.44 & 4.95 & & \\
\hline \multirow{2}{*}{$\begin{array}{l}\text { Autoconcepto } \\
\text { Moral-Ético }\end{array}$} & Primarios y & 69.30 & 4.87 & \multirow{2}{*}{66.50} & \multirow{2}{*}{.48} & 72.25 & 5.73 & \multirow{2}{*}{7.50} & \multirow{2}{*}{.10} \\
\hline & Universitarios & 67.50 & 3.91 & & & 67.44 & 4.64 & & \\
\hline \multirow{2}{*}{$\begin{array}{l}\text { Autoconcepto } \\
\text { Personal }\end{array}$} & Primarios y & 64.70 & 4.59 & \multirow{2}{*}{40.50} & \multirow{2}{*}{.03} & 67.00 & 1.63 & \multirow{2}{*}{9.50} & \multirow{2}{*}{.19} \\
\hline & Universitarios & 69.31 & 5.36 & & & 70.22 & 5.14 & & \\
\hline \multirow{2}{*}{$\begin{array}{l}\text { Autoconcepto } \\
\text { Familiar }\end{array}$} & Primarios y & 67.20 & 6.33 & \multirow{2}{*}{65.50} & \multirow{2}{*}{.45} & 68.00 & 9.05 & \multirow{2}{*}{61.50} & \multirow{2}{*}{.82} \\
\hline & Universitarios & 69.13 & 5.51 & & & 67.44 & 5.72 & & \\
\hline \multirow{2}{*}{$\begin{array}{l}\text { Autoconcepto } \\
\text { Social }\end{array}$} & $\begin{array}{l}\text { Primarios y } \\
\text { Secundarios }\end{array}$ & 68.00 & 4.64 & \multirow{2}{*}{74.00} & \multirow{2}{*}{.77} & 68.25 & 4.99 & \multirow{2}{*}{17.50} & \multirow{2}{*}{.94} \\
\hline & Universitarios & 67.31 & 5.19 & & & 67.89 & 4.54 & & \\
\hline
\end{tabular}

Nota: Diferencia significativa en $p<.05$ 
Al indagar en el nivel de estudios en el grupo de hombres con RP se hallaron diferencias en el factor personal entre los que tenían estudios universitarios $(U=40.50 ; p=0.03)$, mostrando puntuaciones más altas frente al grupo que poseían estudios primarios y medios.

En el grupo de hombres sin afectación no se hallaron diferencias en ningún factor, constatándose incluso peores puntuaciones en aquellos con estudios universitarios. En el resto de factores no hubo diferencias significativas (Tabla 4).

\section{Discusión y Conclusiones}

En este artículo se ha tratado de dar respuesta a los dos objetivos planteados en la presente investigación. El primero buscaba determinar intra-sexos la posible existencia de diferencias en algún factor del autoconcepto en población afectada por RP frente a mujeres y hombres sin afectación. Los resultados han constatado que tanto las mujeres como los hombres con RP manifestaban dificultades en el ámbito físico cuando se comparaban con población sin afectación. Si se tiene en consideración las restricciones asociadas a la problemática visual, que cursa con pérdida de visión periférica, déficit de visión durante la noche, o en condiciones de poca iluminación; problemas de adaptación a los cambios en la iluminación, o en la discriminación de colores (Rundquist, 2004); parece coherente concluir que estas restricciones podrían explicar sus limitaciones en este factor del autoconcepto, así como sus puntuaciones más bajas en todos los factores restantes, aunque no llegaran a ser significativas.

Si bien una investigación anterior puso de relieve que las mujeres con RP se percibían mejor en el factor físico frente a hombres también afectados (López-Justicia, Fernández-Castillo, et al., 2011), los datos del presente estudio dejan entrever que tanto hombres como mujeres de entre 18 y 30 años se sienten peor en este factor cuando se comparan con población sin afectación. Este resultado parece previsible si se tiene en cuenta las características fisiológicas de la problemática visual y la fuerte influencia cultural que ejerce una sociedad que sobrevalora la belleza física y el culto al cuerpo armónico y saludable (Hahn, 1996), por encima de otras cualidades. A pesar de no haber encontrado investigaciones con las que poder contrastar estos resultados, una posible explicación a los mismos la proporciona la teoría de la Comparación Social propuesta por Clark (1994). En ésta se sugiere la tendencia de las personas a relacionar sus características tales como el estado de salud, su apariencia, etc., con las de otros reales o imaginarios; lo que podría influir en el estado de ánimo, en la autoevaluación, o en la satisfacción personal consigo mismo.

Respecto al segundo objetivo (dilucidar diferencias intra-sexos asociadas al nivel de estudios en algún factor del autoconcepto), los resultados del análisis efectuado en el grupo de mujeres con RP constataban que el nivel de estudios desempeñaba un papel a tener en cuenta en el autoconcepto físico; siendo aquellas con estudios universitarios las que puntuaban más alto, no solo en éste sino en todos los factores evaluados, aunque solo aquél llegara a ser significativo. Estos datos dejan entrever que la formación académica, y el sentimiento de competencia o estatus reconocido asociado a ella, parece ser un aspecto relevante vinculándose a puntuaciones más altas en autoconcepto, al igual que se ha sugerido en población sin discapacidad (Goñi et al., 2015). Una mayor formación académica suele ir asociada a mayor capacidad, competencia, oportunidades, habilidades, etc., siendo una experiencia favorecedora del sentimiento de valía personal (López-Justicia et al., 2011). Ello podría explicar la mejor aceptación de su cuerpo, de la discapacidad visual y el mayor interés por su aspecto y forma física. 
Resultado en línea con el obtenido por López-Justicia et al. (2011) y coincidente, en parte, con el encontrado por Martinez y Sewell (1996), en un estudio llevado a cabo con alumnado con discapacidad visual. Los resultados se ofrecen alentadores, puesto que las mujeres con discapacidad suelen ser doblemente vulnerables, enfrentándose a diferentes estereotipos y situaciones de desigualdad (LópezJusticia y Chacón-López, 2015; Vaca, Cordoba, Rosero, Gómez-Benito, Escobar y Lucas-Carrasco, 2011).

Cuando se lleva a cabo la comparación en el grupo de hombres con RP se constata que quienes tienen estudios universitarios se perciben mejor en el factor personal, mostrando una autopercepción más positiva de sus valores interiores, de su sentimiento de adecuación como persona, independientemente de su físico y de sus relaciones con los demás, de acuerdo con Fitts (1965) y Fitts y Warren (1996). En los restantes factores no se obtuvieron diferencias, aunque se detectaron puntuaciones mayores en el factor físico y familiar (ver Tabla 4).

En España no ha sido frecuente hasta hace pocos años que personas con discapacidad accedieran a estudios medios o universitarios, siendo el porcentaje de estudiantes universitarios solamente de 1.5 en el año 2018 (Fundación Universia y CERMI, 2018), a pesar de contemplarse el derecho de todas las personas a la educación y a lo largo del ciclo vital en el II Plan de Acción Nacional para las personas con Discapacidad (2003-2007), así como en el III Plan (2009-2012). Contrariamente a esta cifra, en el presente trabajo se constata que casi la mitad de la muestra con RP posee niveles educativos medios o superiores, siendo las personas con estudios universitarios las que mejor y más satisfechas parecen encontrarse. Sin duda, es un dato interesante, que podría explicarse por el sentimiento de competencia que experimentan, tal vez por haber sido capaces de cursar este nivel de estudios a pesar de las limitaciones que conlleva la problemática visual. Aunque también podría hipotetizarse que las personas que han accedido a la universidad, posiblemente, lo hayan logrado gracias al mayor apoyo ofrecido por sus familiares, o por haber vivido unas experiencias más favorables en su entono. La relación familiar y la ayuda proporcionada por los familiares se ha asociado con una mejor adaptación a la pérdida de visión (Reinhardt, 2001), con reducción de los niveles de depresión (Nunes, Munhoz, y Ferrari, 2014), y con influencia positiva en el autoconcepto físico y personal (Chacón-López y López-Justicia, 2016). No obstante, conviene recordar que la familia puede actuar tanto como apoyo (Goñi et al., 2015; Nunes, 2014), como ser un obstáculo en el desarrollo personal de afectados por discapacidad, dependiendo de sus actitudes hacia éstos (Vaca et al., 2011).

Si bien es cierto que el tipo de análisis estadístico llevado a cabo en la presente investigación no permite probar relaciones de causalidad, sí se puede concluir que el nivel de estudios en población con RP de entre 18 y 30 años tiene un valor digno de consideración, avalado por el hecho de que si se observan las Tablas 2 y 4 se comprueba que tanto las mujeres como los hombres con estudios superiores obtienen puntuaciones mayores en casi todos los factores de autoconcepto. Sin embargo, en población sin afectación no se observan diferencias asociadas al nivel de estudios en ningún caso, contrariamente a lo sugerido por Goñi et al. (2015).

Las implicaciones del presente trabajo radican en que proporcionan pistas que pueden ayudar a los profesionales de la orientación y la psicopedagogía en la toma de decisiones relativa a la actuación con personas afectadas por RP, especialmente en una época del ciclo vital en la que deben dilucidar acerca de su futuro. Concretamente, se puede concluir que uno de los aspectos que se deben potenciar en este colectivo es el referido a la mejora del autoconcepto físico, con el fin de contrarrestar y paliar parte de los efectos de una enfermedad degenerativa como la presente, sobre su bienestar psicológico; puesto que altos niveles de autoconcepto se vinculan con el estado emocional (Chacón-López y LópezJusticia, 2016) y se relacionan con el éxito para enfrentarse a las consecuencias de la discapacidad, con una actitud positiva hacia la vida y mayor participación y compromiso social (Smith et al., 2003). Conclusión a la que se llega, fundamentalmente, tras observar las diferencias entre hombres y mujeres con RP frente a otros sin ella. Ciertamente, se podría argumentar que estas diferencias solo son 
significativas en el factor físico, pero una mirada a las Tablas 1 y 3 permite observar que sus puntuaciones son más bajas en casi todos los factores evaluados; tal vez una muestra mayor hubiera permitido detectarlas. Aun asumiendo esta limitación, los resultados dejan entrever que la competencia percibida por las personas afectadas (vinculada, sobre todo, a la formación académica) se asocia a puntuaciones más altas en algunos factores del autoconcepto, derivándose de ahí que la formación académica parece tener un papel importante en su valoración personal.

Esto nos lleva a sugerir a los profesionales de la orientación y la psicopedagogía que abordan la actuación con estos jóvenes la necesidad de prestar más atención al desarrollo del autoconcepto desde los primeros años tras el diagnóstico de la enfermedad. Con este fin se sugiere identificar los puntos fuertes en los que cada cual destaque reforzándolos y resaltándolos, así como detectar los aspectos más deficitarios para entrenarlos, en aras de favorecer el sentimiento de valía puesto que se ha vinculado la percepción de competencia personal a puntuaciones más altas en autoconcepto (Martinez y Sewel, 1996). Propuesta que se sustenta no solo en los datos obtenidos en la presente investigación sino en otras llevadas a cabo con población juvenil y de mayor edad (Chacón-López y López-Justicia, 2016; López-Justicia et al., 2011).

Si bien no se puede obviar que la RP tiene un carácter progresivo y crónico, por lo que actualmente es imposible impedir su avance, sí es posible adoptar algunas medidas de ayuda psicopedagógica (en forma de adaptaciones de materiales, recursos tecnológicos y apoyo personal) que contrarresten el progreso de la enfermedad y promover el bienestar emocional de esta población para prevenir posibles trastornos asociados (Augestad, 2017a; Chacón-López y López-Justicia, 2016). Con ello se pretende que continúen participando activamente en todos los ámbitos de la vida educativa, personal, social, formativa, laboral, etc., propiciando de este modo un desarrollo óptimo.

A pesar del interés que este trabajo puede tener en la causa de personas que presentan esta problemática, también posee como limitación (aparte de la señalada y el tamaño reducido de la muestra) el número desigual entre hombres y mujeres con RP, por lo que las conclusiones deben ser analizadas con cautela. Aun así, conviene subrayar que el trabajo aborda la problemática de personas que presentan un trastorno que no tiene una incidencia muy alta (provocando por ello que se corra el riesgo de olvidar sus dificultades, necesidades y medidas psicopedagógicas que requieren), y se centra en el análisis de una sola patología degenerativa retiniana.

\section{Referencias}

Augestad, L.B. (2017a). Mental health among children and young adults with visual impairment: a systematic review. Journal of Visual Impairment y Blindness, 111(5), 411-425.

Augestad, L.B. (2017b). Self-concept and self-esteem among children and young adults with visual impairment: A systematic review. Cogent Psychology, 4, 1-13. doi: 10.1080/23311908.2017.1319652

Al-Zyoudi, M. (2007). Gender differences in self-concept among adolescents with low vision. International Journal of Special Education, 22, 132-136. 
Chacón-López, H., y López-Justicia, M.D. (2016). Relación entre autoconcepto y nivel de depresión en personas con retinosis pigmentaria. Anales de Psicología, 32(3), 820-827. doi: 10.6018/analesps.32.3.218081

Chacón-López, H., López-Justicia, M.D., Fernández, C., Chacón, A., y Polo, T. (2013). Emotional state of family members of adults with retinal degeneration. Anales de Psicología, 29(3), 772-778. doi: 10.6018/analesps.29.3.145181

Chacón-López, H., López-Justicia, M.D. y Vervloed, P.J. (2014). Psychological and Educational recommendations for working with young people with Retinitis Pigmentosa. School Psychology International Journal, 35(4), 357-369. doi: 10.1177\%2F0143034313476398

Clark, R.D. (1994). The role of censorship in minority influence. European Journal of Social Psychology, 24(3), 331-338. doi: 10.1002/ejsp.2420240303

Coll, C. (2002). Constructivismo y Educación. En C. Coll, J. Palacios, y A. Marchesi, Desarrollo Psicológico y educación, Vol. 2, Psicología de la educación escolar. Madrid: Alianza Editorial.

Datta, P., y Talukdar, J. (2016). The impact of visión impairment on students' self-concept. International Journal of Inclusive Education, 20, 659-672. DOI: 10.1080/13603116.2015.1111441

Fernández, E. (2007). Retinosis Pigmentaria: Preguntas y respuestas. Elche: Cátedra Bidons Egara.

Fernández, C., López-Justicia, M.D. y Polo, M.T. (2007). Discapacidad visible y no visible: diferencias en el autoconcepto. Estudios de Psicología, 28(3), 359-368. doi: 10.1174/021093907782506498

Fernández-Sierra, J. y Santos-Bocero, G.L. (2014). Orientar las transiciones del alumnado inmigrante: más que un reto multiprofesional. Revista Española de Orientación y Psicopedagogía, 25 (2), 823. doi: 10.5944/reop.vol.25.num.2.2014.13517

Fitts, W.H. (1965). Tennessee Self-Concept Scale: Manual. Tennessee: Counselor Recording and Press.

Fitts, W.H., y Warren, W.L. (1996). Tennessee Self-Concept Scale: Manual. Western Psychological Services.

Fuentes, M.C., García, J.F., Gracia, E., y Lila, M. (2011). Autoconcepto y ajuste psicosocial en la adolescencia. Psicothema, 23(1), 7-12.

Fundación Universia y CERMI. (2018). Universidad y Discapacidad. IV Estudio sobre el grado de inclusión del sistema universitario español respecto de la realidad de la discapacidad. Madrid: Fundación Universia.

Fuhr, P.S.W., Liu, L., y Kuyk, T.K. (2007). Relationships between feature search and mobility Performance in persons with severe visual impairment. Optometry and Vision Science, 84, 393400. doi: 10.1097/OPX.0b013e31804f5afb

Garaigordobil, M., y Bernarás, E. (2009). Self-concept, Self-esteem, personality traits and psychopathological symptoms in adolescents with and without visual impairment. The Spanish Journal of Psychology, 12(1), 149-160. doi: 10.1017/S1138741600001566

Goñi, E., Esnaola, I., Rodríguez, A., y Camino, I. (2015). Personal self-concept and satisfaction with life in adolescence, youth and adulthood. Psicothema, 27(1), 52-58. doi: 10.7334/psicothema2014.105 
Hahn, H. (1996). Embodied Differences. A publication of the Amputee Coalition of America. InMotion, 6(4). Extraído de: http://www.amputee-coalition.org/inmotion/aug_sep_96/embodied_dif.html

Halder, S., y Datta, P. (2012). An exploration into self-concept: a comparative analysis between the adolescents who are sighted and blind in India. British Journal of Visual Impairment, 30, 31-41. doi: 10.1177\%2F0264619611428202

Harter, S. (1999). The construction of self: A developmental perspective. New York: The Guilford Press.

Jessup, G.M., Bundy, A.C., Hancock, N., y Broom, A. (2018). Being noticed for the way you are: social inclusion and high school students with vision impairment. British Journal of Visual Impairment, 36, 90-103. doi: 10.1177\%2F0264619616686396

Kiser, A., y Dagnelie, G. (2008). Reported effects of non-traditional treatments and complementary and alternative medicine by Retinitis Pigmentosa patients. Clinical and Experimental Optometry, 91(2), 166-176. doi: 10.1111/j.1444-0938.2007.00224.x

López-Justicia, M.D., y Chacón-López, H. (2015). Cambios emocionales asociados a la edad y género en personas con degeneración retiniana. Revista Argentina de Clínica Psicológica, 24(1), 57-66.

López-Justicia, M.D., y Nieto, I. (2006). Self-concept of Spanish young adults with Retinitis Pigmentosa. Journal of Visual Impairment and Blindness, 100(6), 366-370.

López-Justicia, M.D., y Pichardo, M.C. (2001). Self-concept and gender in Spanish low-vision adolescents. Visual Impairment Research, 3(1), 7-16.

López-Justicia, M.D., Fernández-Castillo, A., Fernández, C., y Polo, M.T. (2011). Age, Educational Level and Gender in Self-concept of People with Retinitis Pigmentosa. Anales de Psicología, 27(2), $292-$ 297.

López-Justicia, M.D., Polo, T. Fernández, C., Chacón-López, H., Díaz-Batanero, C., y Chacón, A. (2011). Depresión y ansiedad en personas con retinosis pigmentaria, sus familiares y un grupo externo. Universitas Psychologica, 10(2), 467-476.

Manitsa, I., Barlow-Brown, F., y Lyons, E. (2019). Self-concept of adolescents with visual impairment. British Journal of Visual Impairment. Extraído de: https://journals.sagepub.com/doi/10.1177/0264619619883900

Martinez, R., y Sewell, K. (1996). Self-concept of adults with visual impairments. Journal of Rehabilitation, 62(2), 55-59.

Moschos, M., Chatzirallis, A., y Chatzirallis, I. (2015). Psychological aspects and depression in patients with retinitis pigmentosa. European Journal of Ophthalmology, 25(5), 459-462. doi: $10.5301 /$ ejo.5000590

Mishra, V., y Singh, A. (2012). A comparative study of selfconcept and self-confidence of sighted and visually impaired children. Excel International Journal of Multidisciplinary Management Studies, 2, 148-156.

Nunes, M., Munhoz, A., y Ferrari, H. (2014). Depression, family support and hopelessness: a correlated study. Universitas Psychologica, 13(2), 693-702. doi: 10.11144/Javeriana.UPSY13-2.dfsh 
Reinhardt, J.P. (2001). Effects of positive and negative support received and provided on adaptation to chronic visual impairment. Applied Developmental Science, 5, 76-85. doi: 10.1207/S1532480XADS0502_3

Rundquist, J. (2004). Low Vision Rehabilitation of Retinitis Pigmentosa. Journal of Visual Impairment and Blindness, 98, 718-724.

Simón Rueda, C., Fernández-Blázquez, M. L., Pérez-de la Merced, H., Márquez Vázquez, C., Echeita Sarrionandia, G. y Moreno Hernández, A. (2019). Entre el derecho a la educación inclusiva y la realidad: Análisis del III Plan de Acción para Personas con Discapacidad de la comunidad de Madrid y propuestas. Revista Española de Orientación y Psicopedagogía, 30 (1), 57-74. doi: 10.5944/reop.vol.30.num.1.2019.25194

Smith, E., Nolen-Hoeksema, S., Fredrickson, B., y Loftus, G. (2003). Introducción a la Psicología. Madrid: Thomson.

Vaca, P., Cordoba, L., Rosero, R., Gómez-Benito, J., Escobar, N., y Lucas-Carrasco, R. (2011). Creencias y prácticas de mujeres con discapacidad frente al ejercicio de sus derechos. Estudios de Psicología, 32(2), 209-226. doi: 10.1174/021093911795978153

Zapata, M.A. (2018). Personal disability identity in Retinitis Pigmentosa. Rehabilitation Psychology, 63(4), 512-520. doi: 10.1037/rep0000238

Fecha de entrada: 26 noviembre 2018

Fecha de revisión: 01 marzo 2019

Fecha de aceptación: 06 marzo 2020 